\title{
CCN3/Nephroblastoma Overexpressed Is a Functional Mediator of Prostate Cancer Bone Metastasis That Is Associated with Poor Patient Prognosis
}

\author{
Matthew Dankner, ${ }^{*}$ Véronique Ouellet, ${ }^{\dagger}$ Laudine Communal, ${ }^{\dagger \ddagger}$ Estelle Schmitt, ${ }^{\dagger \ddagger}$ Dru Perkins, ${ }^{*}$ Matthew G. Annis, ${ }^{*}$ \\ Véronique Barrès, ${ }^{\dagger \ddagger}$ Christine Caron, ${ }^{\dagger \dagger}$ Anne-Marie Mes-Masson, ${ }^{\dagger \dagger \S}$ Fred Saad, ${ }^{\dagger \neq \Phi}$ Peter M. Siegel, ${ }^{*}$ and the Canadian Prostate \\ Cancer Biomarker Network
}

\begin{abstract}
From the Goodman Cancer Research Centre, * Department of Medicine, McGill University, Montréal; the Centre de Recherche du Centre Hospitalier de l'Université de Montréal, ${ }^{\dagger}$ Montréal; the Institut du Cancer de Montréal, ${ }^{\ddagger}$ Montréal; and the Departments of Medicine, ${ }^{\S}$ and Surgery, ${ }^{\circledR}$ Université de Montréal, Montréal, Québec, Canada
\end{abstract}

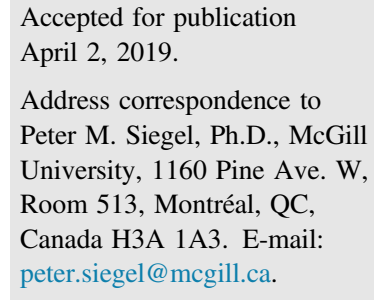

\begin{abstract}
Prostate cancer $(\mathrm{PC})$ commonly metastasizes to the bone, resulting in pathologic fractures and poor prognosis. CCN3/nephroblastoma overexpressed is a secreted protein with a known role in promoting breast cancer metastasis to bone. However, in PC, CCN3 has been ascribed conflicting roles; some studies suggest that CCN3 promotes PC metastasis, whereas others argue a tumor suppressor role for CCN3 in this disease. Indeed, in the latter context, CCN3 has been shown to sequester the androgen receptor (AR) and suppress AR signaling. In the present study, we demonstrate that CCN3 functions as a bone-metastatic mediator, which is dependent on its C-terminal domain for this function. Analysis of tissue microarrays comprising $>1500$ primary PC patient radical prostatectomy specimens reveals that CCN3 expression correlates with aggressive disease and is negatively correlated with the expression of prostate-specific antigen, a marker of AR signaling. Together, these findings point to $\mathrm{CCN} 3$ as a biomarker to predict $\mathrm{PC}$ aggressiveness while providing clarity on its role as a functional mediator of PC bone metastasis. (Am J Pathol 2019, 189: 1451-1461; https://doi.org/10.1016/ j.ajpath.2019.04.006)
\end{abstract}

Prostate cancer (PC) induces mortality by metastasizing to distant organs. Most commonly, PC colonizes the bone, resulting in pathologic fractures and spinal cord compression in the case of vertebral metastases. ${ }^{1}$ Prognostic biomarkers are urgently needed to predict disease aggressiveness and distant metastasis. Biomarkers can help determine the adequate scope of treatment, which can include several modalities, such as surgery, radiation therapy, androgen deprivation therapy, and chemotherapy. ${ }^{2}$

CCN3 (alias nephroblastoma overexpressed) is a founding member of the $\mathrm{CCN}$ family of secreted matricellular proteins [cysteine-rich angiogenic inducer 61 (CYR61), connective tissue growth factor, and nephroblastoma overexpressed]. ${ }^{3,4}$ This family of proteins has been implicated in a broad range of biological processes, including cell proliferation, motility, invasiveness, and angiogenesis. ${ }^{5}$ In the context of PC, CCN3 has recently been proposed to function as a tumor suppressor. ${ }^{6,7} \mathrm{CCN} 3$ expression is negatively regulated by the androgen receptor (AR) and, when expressed, CCN3 has been shown to sequester and impair AR signaling. ${ }^{6,7}$ In contrast,

Supported by a Prostate Cancer Canada discovery grant D2013-31 (P.M.S.), the McGill MD/PhD program (M.D.), the McGill Anatomy and Cell Biology Department John Bergeron Scholarship (M.D.), and the McGill Integrated Cancer Research Training Program (M.D.). F.S. holds the Montreal University Research Chair in Prostate Cancer. V.O., A.-M.M.M., F.S., E.S, L.C., C.C., and V.B. are researchers of the Centre de Recherche du Centre Hospitalier de l'Université de Montréal, which receives support from the Réseau de Recherche sur le Cancer of the Fonds de la Recherche Québec-Santé (FRQS; Canada). P.M.S. is a McGill University William Dawson Scholar. Biobanking was done in collaboration with the FRQS, which is affiliated with the Canadian Tumor Repository Network. Construction of the tissue microarrays was supported by funding from the Terry Fox Research Institute (Canada).

M.D. and V.O. contributed equally to this work.

Disclosures: None declared. 
earlier studies demonstrated that $\mathrm{CCN} 3$ is highly expressed in aggressive prostate cancer cell lines ${ }^{8}$ and increases prostate cancer cell motility and metastasis through a pathway involving intercellular adhesion molecule 1 or by inducing an epithelial-to-mesenchymal transition. ${ }^{9,10}$ In addition to these tumor cell intrinsic effects, secreted CCN3 has been shown to simultaneously alter the bone microenvironment by promoting osteolysis and polarizing macrophages toward the M2 phenotype, promoting angiogenesis and tumor progression of prostate cancer. $^{11,12}$

CCN3 is composed of four domains: an insulin-like growth factor binding domain, a von Willebrand factor type $\mathrm{C}$ domain, a thrombospondin type-1 repeat domain, and a cysteineknot-containing C-terminal (CT) domain. ${ }^{5}$ The $\mathrm{CT}$ domain is thought to be crucial to CCN3's role in cell proliferation ${ }^{13}$ and is known to bind growth factors and heparan sulfate proteoglycans, in addition to activating Notch signaling. ${ }^{5,14}$ The CT domain also promotes homodimerization/heterodimerization of CCN family molecules. ${ }^{5,15}$

CCN3 plays an important role in breast cancer bone metastasis by modulating osteoblast and osteoclast differentiation. ${ }^{16}$ Herein, these findings were extended by clarifying CCN3's role as a functional mediator of PC bone metastasis, which depends on its CT domain for the enhanced formation of bone metastases. This study also shows that high CCN3 expression in primary PC specimens is correlated with biochemical and bone-specific relapse. These findings suggest that $\mathrm{CCN} 3$ represents a strong candidate for prospective studies as a PC biomarker.

\section{Materials and Methods}

\section{Cell and Tissue Culture}

PC cell lines were acquired from ATCC (Manassas, VA). PC3, 22RV1, DU145, LNCaP, LNCaP C4, and LNCaP C42 cells were grown in RPMI 1640 medium supplemented with $10 \%$ fetal bovine serum. LNCaP C4-2 cells were infected with pHIV-Luc-ZsGreen lentivirus (Addgene, Watertown, Massachusetts; plasmid number 39196) and retrovirus to express constructs within the PQX1B vector backbone [vector control, wild-type $\mathrm{CCN} 3\left(\mathrm{CCN} 3{ }^{\mathrm{WT}}\right)$, and $\mathrm{CCN} 3$ that lacks the $\mathrm{CT}$ domain $(\mathrm{CCN} \Delta \mathrm{CT})]$. Retroviral infected cells were grown in the same media supplemented with $10 \mu \mathrm{g} / \mathrm{mL}$ blasticidin as a selection agent.

\section{Migration and Invasion Assays}

PC cells $\left(1 \times 10^{5}\right)$ were suspended in serum-free medium, incubated in the top chamber of $8-\mu \mathrm{m}$ pore cell culture inserts (Falcon; Corning, Corning, NY), and allowed to migrate through the pores for 24 hours toward complete medium. Invasion assays were performed using the same protocol, with the addition of $4 \%$ Matrigel coating the top of the insert. After 24 hours, cells were fixed with $10 \%$ formalin and stained with crystal violet (Sigma-Aldrich, St.
Louis, MO). The top of the chamber was wiped with a cotton swab to remove residual cells from the top of the filter and reveal the cells that traveled through to the bottom of the insert. Five representative images were taken of the bottom chamber of the insert, and positive pixel quantification was performed using Scion Imaging Software version 4.0 (NIH, Bethesda, MD). Three independent experiments were performed for a total of 12 independent wells for each experimental arm. A $t$-test was used to compare migration and invasion of experimental arms.

\section{Immunoblot Analysis}

Samples were prepared from cell lysates or conditioned media diluted in Tris-NaCl-EDTA (TNE) lysis buffer. Conditioned medium was prepared by seeding tumor cells to $70 \%$ confluence, followed by 48 -hour incubation with serumfree medium that was used as conditioned medium. All immunoblots were performed in triplicate, as previously described. ${ }^{17}$ Representative immunoblots are shown. The antibodies used for immunoblotting were as follows: CCN3 (Abcam, Cambridge, UK; catalog number 137677), V5 (Invitrogen, Carlsbad, CA; catalog number 46-0705), prostate-specific antigen (PSA; Ventana, Oro Valley, AZ; catalog number ER-PR8), AR (Cell Signaling; catalog number 5153), laminin A/C (Cell Signaling, Danvers, MA; catalog number 4777), E-cadherin (Cell Signaling; catalog number 3195), glyceraldehyde-3-phosphate dehydrogenase (Millipore, Burlington, MA; catalog number MAB474), and $\alpha$-tubulin (Sigma-Aldrich; catalog number T9026).

\section{RNA Extraction, cDNA Synthesis, and Quantitative PCR}

RNA extraction and cDNA synthesis were performed, as previously described. ${ }^{17}$ Quantitative PCR was performed using SYBR Green Master Mix (Roche Diagnostics, Basel, Switzerland) with $10 \mathrm{mmol} / \mathrm{L}$ primer pair stock. CCN3 and $\beta$-actin specific primers were used for PCR amplification. Experiments were performed in triplicate.

\section{Enzyme-Linked Immunosorbent Assay}

Nephroblastoma overexpressed/CCN3 enzyme-linked immunosorbent assays (R\&D Systems, Minneapolis, MN; catalog number DY1640) were performed, according to the manufacturer's instructions. Conditioned medium was harvested from cells (approximately $70 \%$ confluence) cultured in serum-free medium for 48 hours. Experiments were performed in triplicate.

\section{Proliferation Assay}

PC cells $\left(1 \times 10^{4}\right)$ were seeded in each well of five 24-well plates. Each day, one plate was removed, stained with crystal violet, and incubated at $570 \mathrm{~nm}$ to determine viable cell concentration. Absorbance was measured at $570 \mathrm{~nm}$ to 
determine viable cell concentration. Experiments were performed in triplicate, and a $t$-test was used to compare proliferation of experimental arms.

\section{Subcellular Fractionation}

Subcellular fractionations were performed, as per manufacturer instructions, using the subcellular protein fractionation kit for cultured cells (Thermo Scientific, Waltham, MA; catalog number 78840). For fractionation experiments, immunoblots were incubated with IR dye secondary antibodies (Licor Inc., Lincoln, NE) and developed with the Odyssey Imager (Licor Inc.).

\section{Intracardiac Injections}

LNCaP C4-2 PC cells $\left(1 \times 10^{6}\right)$ were injected into the left cardiac ventricle of male NCR nude mice, aged 5 to 6 weeks, under isoflurane-induced anesthesia, as previously described. ${ }^{18}$ Only mice with full-body bioluminescence signal immediately after injection were included in subsequent analyses. All animal studies and protocols were approved by the McGill Comparative Medicine and Animal Resources Centre, in accordance with guidelines established by the Canadian Council on Animal Care.

\section{Bioluminescence Measurements}

Mice were injected with $50 \mu \mathrm{L}$ of in vivo luciferase reagent (Perkin Elmer Inc., Waltham, MA) and imaged using the IVIS-100 imaging system (Perkin Elmer Inc.) with Living Image software version 4.3 (Perkin Elmer Inc.). PC cell growth was monitored biweekly and was plotted as a ratio of flux (photons/second)/the initial whole-body bioluminescent signal. A $t$-test was used to compare luminescence intensities between experimental arms.

\section{$\mu \mathrm{CT}$ Analysis}

Ex vivo micro computed tomography $(\mu \mathrm{CT})$ scans (Skyscan 1178; Bruker Inc., Kontich, Belgium) were performed on isolated bones fixed for 24 hours in $4 \%$ paraformaldehyde 8 weeks after intracardiac injection. Scans were reconstructed and analyzed for bone volume and bone mineral density using NRecon, Data Viewer, TConV, CTAn, and CTVol software version 1 (Bruker Inc., Kontich, Belgium). A $t$-test was used to compare bone mineral density between experimental arms.

\section{TMA Construction}

Tissue microarray (TMA) TF123 was constructed at the Centre de Recherche du Centre Hospitalier de l'Université de Montréal. This series is composed of 300 radical prostatectomy specimens from patients included in the Centre de Recherche du Centre Hospitalier de l'Université de Montréal-RRCancer prostate cancer biobank. For each patient, two cores $(0.6 \mathrm{~mm}$ thick) of tumor and two cores of benign gland were extracted from formalin-fixed, paraffin-embedded radical prostatectomy specimens and arrayed on receiver blocks. On the basis of clinical data review, 15 cases were excluded from the analysis because of preoperative treatments. Thus, a total of 285 PC treatment-naïve specimens were used for this study.

The Test-TMA and Validation-TMA series were accessed via the Canadian Prostate Cancer Biomarker Network $(\mathrm{CPCBN}) .{ }^{19}$ Data from test and validation TMAs were combined to form what is referred to hereafter as the CPCBN TMA. This resource contained 1512 radical prostatectomy specimens from treatment-naïve PC patients enrolled at five different institutional prostate cancer biobanks: Centre de Recherche du Centre Hospitalier de l'Université de Montréal, Research Institute of the McGill University Health Center, Centre Hospitalier Universitaire de Québec-Université Laval, University Health Network, and the Vancouver Prostate Centre. For each patient, 0.6-mm cores (three to four of tumor tissue and one to two of benign glands) were extracted from blocks and transferred to a receiver block. All patients included in the TF123 and the CPCBN TMAs signed an informed consent form to contribute tissue to the indicated biobanks. In addition, each biobank received approval from its local ethics review board for inclusion of samples into the CPCBN resource.

\section{Tissue Microarray Staining and Analysis}

Immunohistochemistry assays were performed on sections ( $4 \mu \mathrm{m}$ thick) of the TF123 TMA using the Benchmark XT autostainer (Ventana Medical Systems, Tucson, AZ) to stain for CCN3 (K19M) and PSA (sc-76380). Stained sections were scanned using a VS-110 microscope with a $20 \times 0.75$ numerical aperture objective and a resolution of $0.3225 \mu \mathrm{m}$ (Olympus Canada Inc., Richmond Hill, ON, Canada). Two observers (Canadian Prostate Cancer Biomarker Network) independently scored the staining for $\mathrm{CCN} 3$ (composite score: intensity and percentage). PSA staining was assessed by digital image analysis (mean intensity of staining in the epithelium) using Visiopharm software version 2017.2.7 (Visiopharm, Hørsholm, Denmark).

In the CPCBN TMA, CCN3 was assessed using an immunofluorescence assay on sections ( $4 \mu \mathrm{m}$ thick). CCN3 was visualized in the red channel (excitation, $649 \mathrm{~nm}$; emission, $666 \mathrm{~nm}$ ); epithelial cell mask (cytokeratin 8/18) was labeled to emit in the green channel (excitation, 490 $\mathrm{nm}$; emission, $525 \mathrm{~nm}$ ); and nuclei were labeled to emit in the blue channel (excitation, $350 \mathrm{~nm}$; emission, $470 \mathrm{~nm}$ ) using DAPI (Molecular Probes, Eugene, OR; D3571). Scanned images were imported to Visiopharm software. Semiautomated analysis protocol packages were established to determine the mean fluorescence intensity of CCN3 specifically in the epithelium. Cores with $<5 \%$ of epithelial cells and damaged cores were discarded. In addition, 6 of 38 TMA blocks were excluded because of an issue with the 
epithelial mask staining. A total of 1259 patient specimens were included in the analyses. To compare all TMAs together, mean fluorescence intensity data were normalized using the ratio of mean fluorescence intensity section/mean fluorescence intensity across all TMA sections. Staining conditions for each antibody are shown in Table 1.

Statistical analyses were performed using SPSS software version 25.0 (SPSS Inc., Chicago, IL). For each patient, the average of the scores obtained from cores with the same histology (tumor or benign) was used for the statistical analyses. For $\mathrm{CCN} 3$, the seventy-fifth percentile was used to dichotomize the data. The correlation was estimated using a nonparametric Spearman correlation test. Biochemical relapse (BCR) - and bone metastasis-free and PC-specific survival curves were plotted using the Kaplan-Meier estimator, and the log-rank test was used to evaluate significant differences. Univariate and multivariate Cox regression analyses were used to estimate the hazard ratios for $\mathrm{CCN} 3$. For multivariate analyses, the serum PSA level before the radical prostatectomy, pathologic staging of the primary tumor (primary tumor 2, 3, or 4), Gleason score category [6,7 $(3+4)$, $7(4+3)$, and $8+]$, and margin status (negative/positive) were included in the model. $P<0.05$ was considered statistically significant.

\section{Tartrate-Resistant Acid Phosphatase Staining and Analysis}

Tartrate-resistant acid phosphatase staining was performed, as previously described. ${ }^{20}$ Osteoclast percentage positivity was determined on whole sections using HALO image analysis software version 2.3 (Indica Labs, Corrales, NM).

\section{Results}

\section{CCN3 Promotes 0steolytic Prostate Cancer Bone} Metastases in a CT Domain-Dependent Manner

A panel of PC cell lines with known propensities to form osteoblastic (bone-forming) or osteolytic (bone-resorbing) bone metastases were analyzed for $\mathrm{CCN} 3$ expression by quantitative RT-PCR, immunoblot, and enzyme-linked immunosorbent assay (Figure 1, A-C). CCN3 expression, by all three approaches, was highest in PC cell lines known to form osteolytic bone metastases in vivo (PC3 and DU145) $)^{21,22}$ and lowest in cell lines known to be less aggressive and form osteoblastic lesions ( $\mathrm{LNCaP}){ }^{21} \mathrm{CCN} 3$ expression was readily detectable in prostate cancer cells that form mixed metastases in vivo (22RV1) (Figure 1, $\mathrm{A}-\mathrm{C}){ }^{23}$

To test the contribution of $\mathrm{CCN} 3$ overexpression in prostate cancer, $\mathrm{LNCaP}$ C4-2 PC cells, a derivative cell line from a prostate cancer lymph node metastasis ( $\mathrm{LNCaP}$ ) grown in vivo in castrated mice, ${ }^{24,25}$ were selected because they have low endogenous $\mathrm{CCN} 3$ levels and form bone metastases after intracardiac injection. ${ }^{24,26}$ LNCaP C4-2 PC cells were infected with pHIV-Luciferase-ZS-Green lentivirus for bioluminescence imaging and subsequently infected with a retrovirus harboring the following: i) a PQX1B vector alone (empty vector), ii) $\mathrm{CCN} 3{ }^{\mathrm{WT}}$, or iii) a $\mathrm{CCN} 3$ mutant lacking the $\mathrm{CT}$ domain $\left(\mathrm{CCN}^{\Delta \mathrm{CT}}\right)$ (Figure 1D). Secreted CCN3 was measured in the conditioned medium derived from the engineered cell lines by enzyme-linked immunosorbent assay (Figure 1E) and immunoblot analysis (Figure 1F). Together, these analyses demonstrated similar levels of $\mathrm{CCN} 3$ expression between $\mathrm{CCN} 3^{\mathrm{WT}}$ and $\mathrm{CCN}_{3}{ }^{\mathrm{CCT}}$ in the engineered $\mathrm{LNCaP} \mathrm{C} 4-2$ cell lines. $\mathrm{LNCaP}$ C4-2 cells expressing $\mathrm{CCN} 3$ did not differ from vector control cells in their migratory $(P=0.51)$ (Supplemental Figure S1A), invasive $(P=0.57)$ (Supplemental Figure S1B), or proliferative $(P=0.25)$ (Supplemental Figure S1C) abilities.

Protein expression of AR and PSA did not differ between cell lines (Supplemental Figure S1D). Cell fractionation experiments revealed similar subcellular distribution of AR between cell lines (Supplemental Figure S1E). Together, this suggests that AR signaling is unaltered when either $\mathrm{CCN} 3^{\mathrm{WT}}$ or $\mathrm{CCN} 3^{\Delta \mathrm{CT}}$ is overexpressed.

Athymic nude (nu/nu) mice were subjected to intracardiac injections with LNCaP C4-2 pHIV-ZS-Green-Luc cells

Table 1 Description of Antibodies and Protocols Used for Immunostaining (IHC and IHF)

\begin{tabular}{|c|c|c|c|c|c|c|c|c|c|}
\hline \multirow[b]{3}{*}{ Marker } & \multirow[b]{3}{*}{ Assay } & \multirow[b]{3}{*}{ Company } & \multirow[b]{3}{*}{ Catalog no. } & \multirow[b]{3}{*}{ Clone } & \multicolumn{5}{|l|}{ Ventana staining } \\
\hline & & & & & \multirow{2}{*}{$\begin{array}{l}\text { Antigen retrieval } \\
\text { Reagent; time, } \\
\text { minutes }\end{array}$} & \multicolumn{2}{|c|}{ Primary antibody } & \multicolumn{2}{|c|}{ Secondary antibody } \\
\hline & & & & & & Dilution & $\begin{array}{l}\text { Time, } \\
\text { minutes }\end{array}$ & Type & Condition \\
\hline CCN3 & $\mathrm{IHC}$ & Noncommercial & NA & K19M & $\mathrm{CC} 2 ; 30$ & 1:1000 & 60 & Rabbit & UVDAB \\
\hline PSA & $\mathrm{IHC}$ & $\begin{array}{l}\text { Santa Cruz } \\
\text { Biotechnology } \\
\text { (Dallas, TX) }\end{array}$ & Sc-7638 & NA & $\mathrm{CC} 1 ; 60$ & $1: 1000$ & 60 & Goat & 1:300, PBS \\
\hline CCN3 & IHF & Abcam & Ab137677 & NA & $\mathrm{CC} 2 ; 30$ & $1: 200$ & 100 & Cy5-rabbit & $1: 250$ PBS-BSA, $1 \%$ \\
\hline $\begin{array}{l}\text { Epithelial mask } \\
\text { (CK8, CK18) }\end{array}$ & IHF & Thermo Scientific & $\begin{array}{c}\text { MA5- } 14428 \\
\text { sc- } 6259\end{array}$ & NA & $\mathrm{CC} 2 ; 30$ & 1:1000 & 100 & A546-mouse & $1: 250$ PBS-BSA, $1 \%$ \\
\hline
\end{tabular}

BSA, bovine serum albumin; CC1/CC2, cell conditioning $1 / 2$ solution (supplied by Ventana Medical Systems); CK, cytokeratin; IHC, immunohistochemistry; IHF, immunohistofluorescence; NA, not applicable; PBS, phosphate-buffered saline; PSA, prostate-specific antigen. 


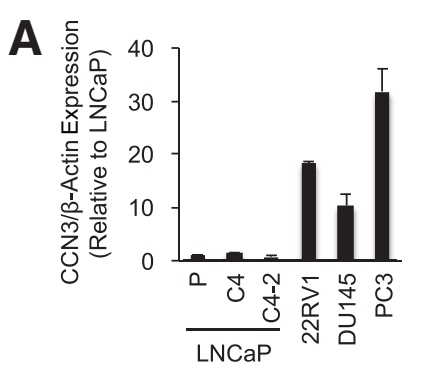

D
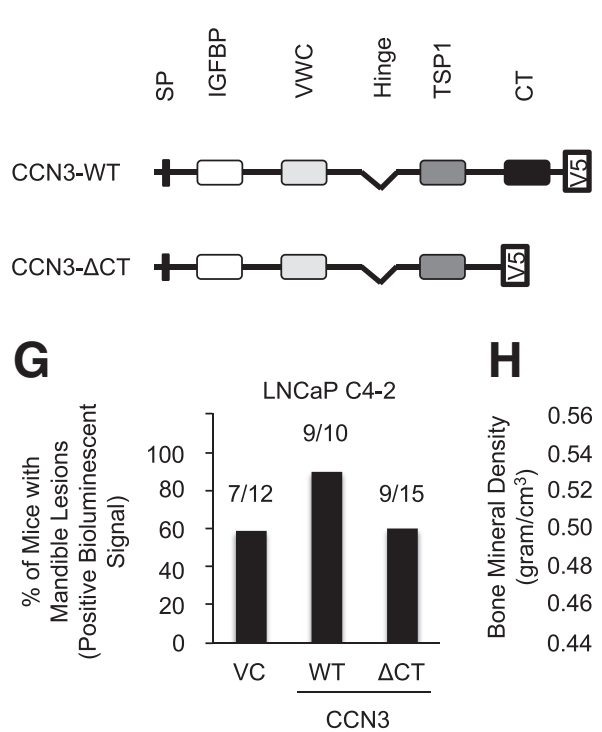

B

H
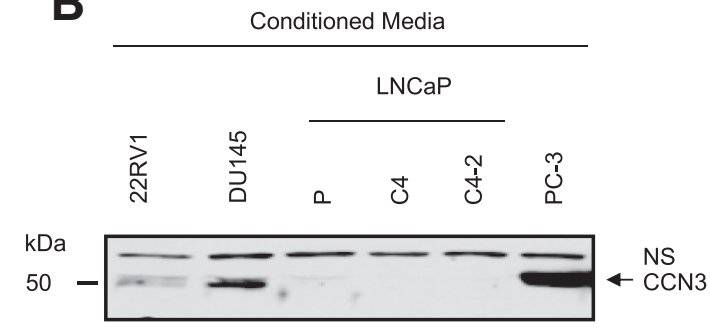

E
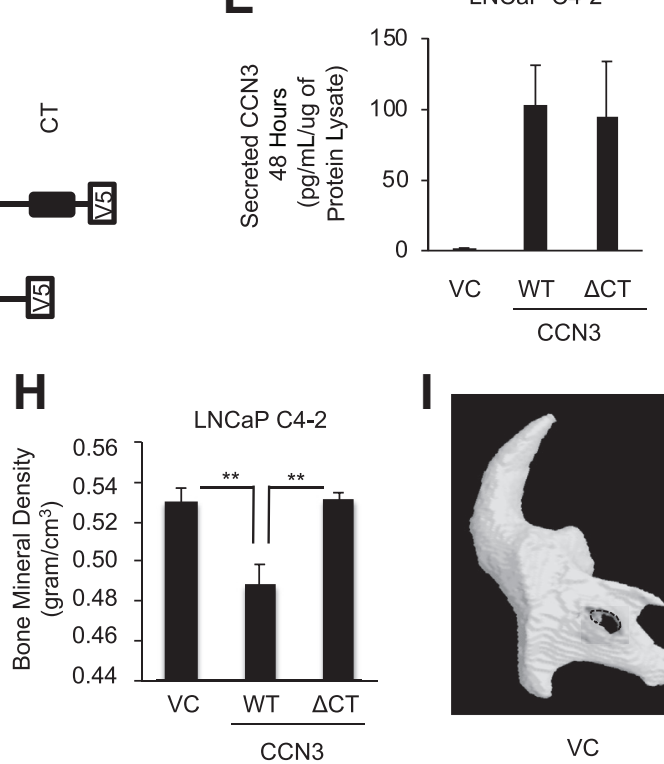
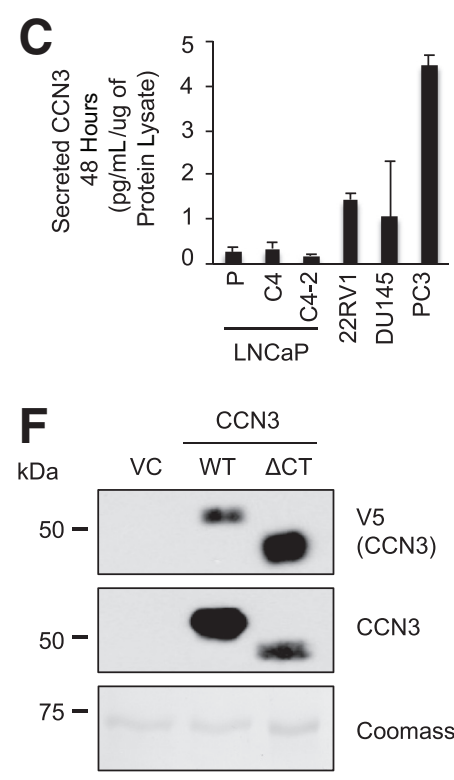

V5 (CCN3) CCN3 Coomassie

Figure 1 CCN3 promotes bone metastasis in a C-terminal cysteine knot domain (CT)-dependent manner. A: mRNA expression of CCN3 in prostate cancer cell lines; data are normalized to $\beta$-actin and expressed relative to LNCaP cells. B: Secreted CCN3 in conditioned media (48 hours) of prostate cancer cells, as determined by immunoblot analysis. The arrow indicates CCN3. C: Secreted CCN3 in conditioned media (48 hours) of prostate cancer cells, as measured by enzyme-linked immunosorbent assay (ELISA). Experiment was performed in triplicate wells. D: Schematic depicting wild-type CCN3 (CCN3 ${ }^{\text {WT }}$ ) with its signal peptide (SP), insulin growth factor-like binding domain (IGFBP), von Willebrand type C domain (VWC), its variable hinge region, its thrombospondin type-1 domain (TSP1), and CT. CCN3 ${ }^{\Delta C T}$ lacks the CT domain. E: Expression of CCN3 ${ }^{\text {WT }}$ and CCN3 ${ }^{\Delta C T}$ in LNCaP C4-2 derived conditioned media, as measured by ELISA. F: Expression of $\mathrm{CCN}^{\mathrm{WT}}$ and $\mathrm{CCN}^{\Delta \mathrm{CT}}$ in $\mathrm{LNCaP}$ C4-2 conditioned media, as measured by immunoblot analysis for the V5 tag at the C-terminus or with antibody specific for CCN3. Coomassie stain for a nonspecific band is used as a loading control. G: Percentage of mice with detectable mandibular bone lesions, as measured by bioluminescence imaging (IVIS) 8 weeks after intracardiac injection of LNCaP C4-2 cells expressing vector control (VC; $58 \%$ ), CCN3 ${ }^{\text {WT }}$ ( $90 \%$ ), and $\mathrm{CCN}^{\mathrm{ACT}}(60 \%)$. H: Micro computed tomography analysis of bone mineral density in mandibles of mice subjected to intracardiac injections with LNCaP C4-2 vector control-, $\mathrm{CCN}^{\mathrm{WT}}{ }^{\mathrm{WT}}$, and $\mathrm{CCN}^{\mathrm{ACT}}$-expressing prostate cancer cells. A $t$-test was used to compare bone mineral density between experimental arms. I: Representative $\mu \mathrm{CT}$ images of mandibles, with areas of osteolysis circumscribed with dashed lines. Data are expressed as means \pm SEM (A, $\mathbf{C}, \mathbf{E}, \mathbf{G}$, and $\mathbf{H}$ ). $n=3$ replicates, for a total of 9 wells per cell line $(\mathbf{C}) ; n=5$ independent experiments, performed in triplicate, for a total of 15 wells per cell line (E). $* * P<0.01$. NS, a nonspecific band used as a loading control.

harboring a vector control or expressing either $\mathrm{CCN} 3{ }^{\mathrm{WT}}$ or $\mathrm{CCN} 3^{\Delta \mathrm{CT}}$. Full-body bioluminescent signal was evident immediately after injection, but by 2 weeks postinjection, luminescent signals from the injected PC cells were undetectable (Supplemental Figure S2, A and B). By 8 weeks postinjection, animals in each cohort exhibited detectable bioluminescence signals (Supplemental Figure S2, A and B). Indeed, 7 of 12 vector control-injected mice (58\%) developed bone metastases when assessed by bioluminescence, compared with 9 of 10 of the mice injected with $\mathrm{CCN} 3{ }^{\mathrm{WT}}$ expressing cells (90\%) and 9 of 15 of the mice injected with $\mathrm{CCN}^{\Delta \mathrm{CT}}$-expressing cells $(60 \%)$ (Figure $1 \mathrm{G}$ ). All bone metastases detected by luminescence imaging were located in the mandible. Growth rates of established mandibular metastases, as measured by bioluminescence imaging, in the $\mathrm{CCN} 3{ }^{\mathrm{WT}}$ cohort did not differ statistically from those in the vector control or $\mathrm{CCN} 3^{\Delta \mathrm{CT}}$ cohorts (vector control versus WT: $P=0.51$; vector control versus $\Delta \mathrm{CT}: P=0.81$ ) (Supplemental Figure S2B). Ex vivo $\mu \mathrm{CT}$ analysis of mandibles revealed a significant decrease in bone mineral density along with substantial bone destruction in the mice injected with $\mathrm{CCN} 3^{\mathrm{WT}}$-expressing PC cells compared with cohorts injected with cells containing the vector control or expressing $\mathrm{CCN}^{\Delta \mathrm{CT}}(P<0.005)$ (Figure $1, \mathrm{H}$ and I). Immunostaining for $\mathrm{CCN} 3$ revealed expression in mandibular lesions from mice injected with $\mathrm{CCN} 3^{\mathrm{WT}}$ - and $\mathrm{CCN} 3^{\Delta \mathrm{CT}}$-expressing cells but not LNCaP C4-2 VC cells (Supplemental Figure S2C). Tartrate-resistant acid phosphatase staining, to specifically 
mark osteoclasts, revealed enhanced osteoclastogenesis in mandibles infiltrated with PC cells expressing CCN3 WT compared with those harboring vector control or a CCN3 mutant that lacks the $\mathrm{CT}$ domain $(P<0.05)$ (Supplemental Figure S3).

\section{CCN3 Expression Is Correlated with Aggressive Disease in Primary PC Specimens}

To assess the value of $\mathrm{CCN} 3$ as a biomarker to predict PC metastasis to bone, $\mathrm{CCN} 3$ expression was analyzed in two independent TMA series that contain primary PC tumors linked to clinical data through the Centre de Recherche du Centre Hospitalier de l'Université de Montréal prostate cancer biobank and the Canadian Prostate Cancer Biomarker Network.
In the first TMA series, TF123 $(n=285$ patients), CCN3 levels were assessed by immunohistochemistry. A composite score was defined according to staining intensity and percentage of cells with the specific intensity (Figure 2A). Patient characteristics are shown in Supplemental Table S1. Using Kaplan-Meier analyses coupled with a log-rank test, it was observed that patients whose PC specimens expressed high levels of $\mathrm{CCN} 3$ ( $>75$ th percentile) experienced a trend toward shortened time to BCR at 5 years $(P=0.081)$ (Figure $2 \mathrm{~B})$ and bone metastasis at 15 years $(P=0.055)$ (Figure $2 \mathrm{C})$. Similar results were seen using a 3 -year cutoff for BCR $(P=0.039)$ and 10 years of follow-up for bone metastasis $(P=0.005)$ (Supplemental Figure S4, A and B). High CCN3 expression correlated with shortened PC-specific overall survival in the TF123 TMA $(P=0.021)$ (Supplemental Figure S4C). In Cox regression analyses, high $\mathrm{CCN} 3$ expression was associated

\section{A}

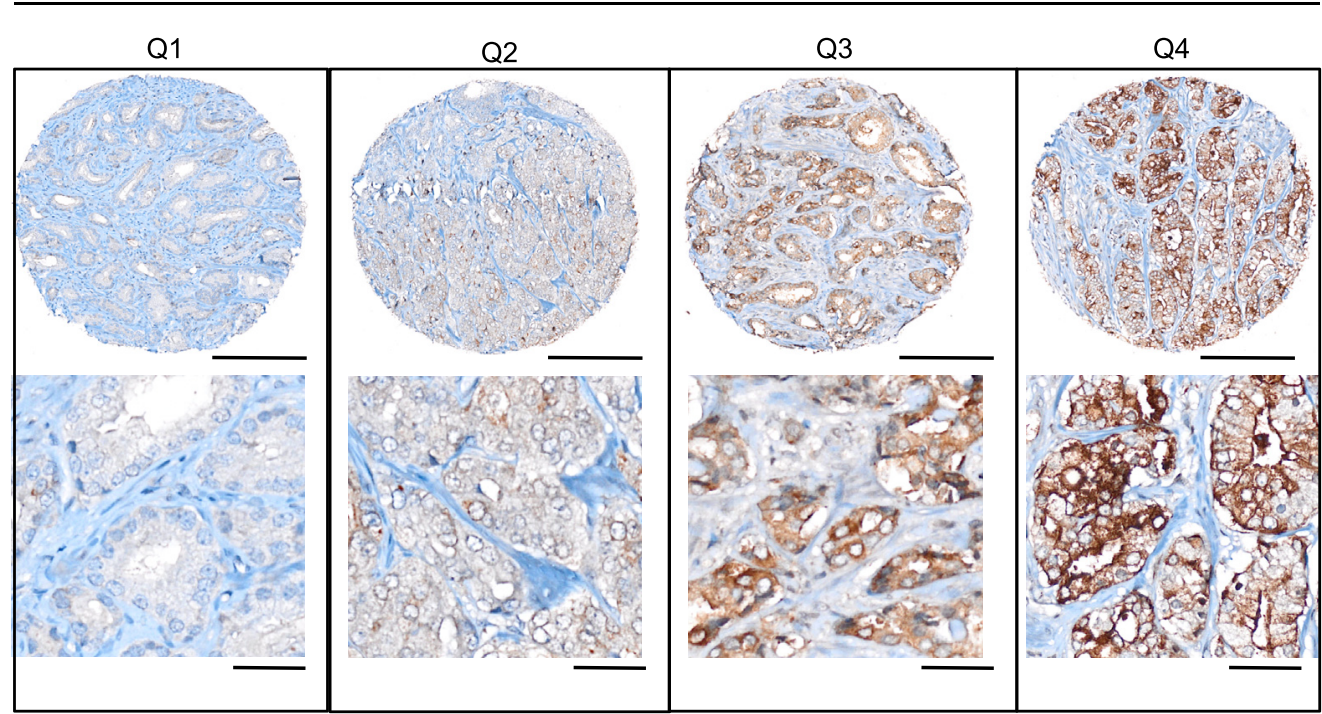

B

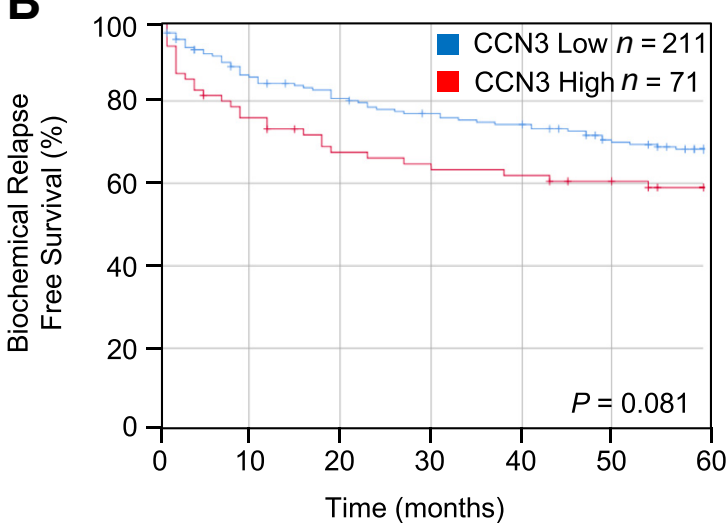

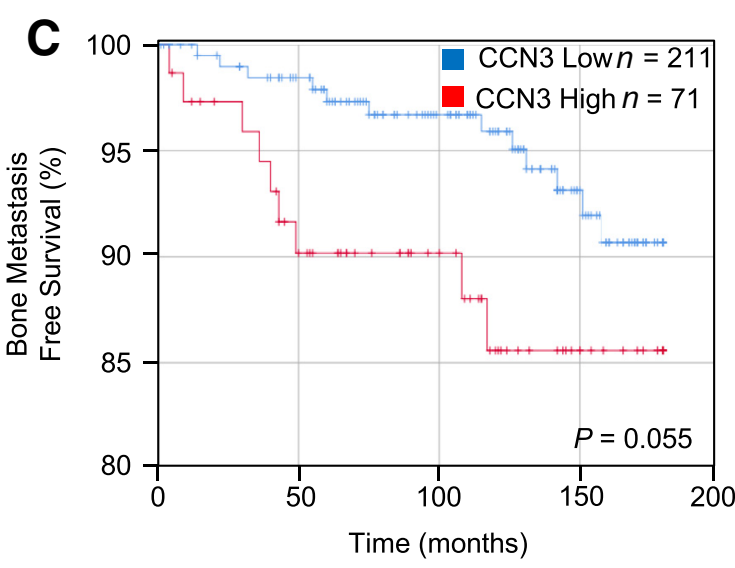

Figure 2 High CCN3 expression by immunohistochemistry (IHC) trends toward increased risk of biochemical relapse and bone metastasis in TF123 tissue microarray (TMA). The TF123 TMA was assessed for CCN3 expression by IHC with antibody K19M. CCN3 high [quartile (Q) 4] represents the 75th percentile of staining intensity, whereas CCN3 low is determined corresponding to the 0 to 75th percentile (Q1 to Q3). Three samples could not be evaluated because of low tumor content and were not included in subsequent analyses. A: Representative images demonstrating staining intensities at the 0, 25th, 50th, and 75th percentiles. B: Tumors with high CCN3 expression (fourth quartile) trend toward correlation with decreased biochemical relapse-free survival at 5 years of follow-up $(P=0.081)$. C: Bone metastasis-free survival at 15 years of follow-up $(P=0.055) . n=285$ primary prostate cancer patient samples $(\mathbf{A}-\mathbf{C})$; $n=71$ (CCN3 high); $n=211$ (CCN3 low). Scale bars: $200 \mu \mathrm{m}$ (A, top row); $50 \mu \mathrm{m}$ (A, bottom row). 
with biochemical relapse at 3 years $[P=0.043$; $\operatorname{Exp}(\mathrm{B})=1.624(95 \% \mathrm{CI}, 1.015-2.598)]$ or trended toward association at 5 years $[P=0.085 ; \operatorname{Exp}(\mathrm{B})=1.469(95 \% \mathrm{CI}$, 0.949-2.274)], although this association was not independent from clinical parameters (Table 2). High expression of CCN3 was significantly associated with the development of bone metastasis at 10 years $[P=0.009 ; \operatorname{Exp}(\mathrm{B})=3.733(95 \% \mathrm{CI}$, 1.389-10.029)] and trended toward statistical significance at 15 years $[P=0.062 ; \operatorname{Exp}(\mathrm{B})=2.279(95 \% \mathrm{CI}$, 0.959-5.414)] (Table 3). In addition, high expression of CCN3 was also significantly associated with the risk of dying from the disease $[P=0.028 ; \operatorname{Exp}(\mathrm{B})=3.003(95 \% \mathrm{CI}$, 1.125-8.012)].

Because CCN3 has been proposed to act as a tumor suppressor in $\mathrm{PC}$ and is regulated by $\mathrm{AR}$ signaling, it was determined whether CCN3 expression is negatively correlated with expression of PSA, a marker of AR signaling. ${ }^{6,7,27}$ TF123 TMA was stained for PSA (Supplemental Figure S5A). CCN3 weakly negatively correlated with expression of PSA (Spearman correlation $=-0.187$; $P=0.002$ ) (Supplemental Figure S5B and Supplemental Table S2). These analyses show, in a large patient cohort, that the regulation of $\mathrm{CCN} 3$ may be mediated by $\mathrm{AR}$ signaling, as has been previously reported. ${ }^{6,7}$

To extend these results in a larger multicenter patient cohort, $\mathrm{CCN} 3$ levels were assessed by immunohistofluorescence in the CPCBN TMA using a different, commercially available CCN3 antibody (Figure 3A). A total of 1259 primary PC specimens were analyzed (Supplemental Table S3). CCN3 levels were categorized using the percentile of the mean fluorescence intensity (Figure 3A). In this TMA, high CCN3 expression (75th percentile) was associated with an increased risk of BCR at 5 years $(P=0.016)$ (Figure 3B) and bone metastasis at 15 years $(P=0.038)$ (Figure $3 C)$. Similar results were seen with BCR cutoff at 3 years $(P=0.027)$ (Supplemental Figure S6A) and bone metastasis at 10 years, which trended toward statistical significance $(P=0.097)$ (Supplemental Figure S6B). In this larger TMA series, when assessed by Cox regression analyses, high expression of CCN3 was significantly associated with BCR at both 3 years $[P=0.013 ; \operatorname{Exp}(\mathrm{B})=1.400(95 \% \mathrm{CI}, 1.072-1.828)]$ and 5 years $[P=0.008 ; \operatorname{Exp}(\mathrm{B})=1.372(95 \% \mathrm{CI}, 1.086-1.735)]$. In addition, this association remains independent from clinical parameters [3 years: $P=0.010 ; \operatorname{Exp}(\mathrm{B})=1.437(95 \% \mathrm{CI}$,

Table 2 Cox Regression Analysis on the TF123 and CPCBN TMA Series for BCR as End Point

\begin{tabular}{|c|c|c|c|c|c|c|c|c|}
\hline \multirow{2}{*}{ BCR as end point } & \multicolumn{4}{|c|}{ Univariate analysis } & \multicolumn{4}{|c|}{ Multivariate analysis } \\
\hline & $\begin{array}{l}\text { Significance } \\
\text { ( } P \text { value })\end{array}$ & $\operatorname{Exp}(B)$ & \multicolumn{2}{|c|}{$\begin{array}{l}95.0 \% \text { CI for } \\
\operatorname{Exp}(B)\end{array}$} & $\begin{array}{l}\text { Significance } \\
(P \text { value })\end{array}$ & $\operatorname{Exp}(B)$ & \multicolumn{2}{|c|}{$\begin{array}{l}95.0 \% \text { CI for } \\
\operatorname{Exp}(B)\end{array}$} \\
\hline \multicolumn{9}{|l|}{ TF123 TMA series } \\
\hline \multicolumn{9}{|l|}{3 Years } \\
\hline Preoperative PSA level & 0.003 & 1.048 & 1.017 & 1.081 & 0.332 & 1.022 & 0.978 & 1.067 \\
\hline Margin status & $<0.001$ & 3.890 & 2.452 & 6.170 & $<0.001$ & 3.016 & 1.817 & 5.007 \\
\hline pTNM & $<0.001$ & 2.934 & 2.122 & 4.056 & 0.031 & 1.626 & 1.047 & 2.527 \\
\hline CCN3 low/high & 0.043 & 1.624 & 1.015 & 2.598 & 0.124 & 1.477 & 0.898 & 2.428 \\
\hline \multicolumn{9}{|l|}{5 Years } \\
\hline Preoperative PSA level & $<0.001$ & 1.061 & 1.033 & 1.089 & 0.039 & 1.039 & 1.002 & 1.077 \\
\hline Gleason score & $<0.001$ & 1.852 & 1.549 & 2.214 & $<0.001$ & 1.490 & 1.210 & 1.835 \\
\hline \multicolumn{9}{|l|}{3 Years } \\
\hline Preoperative PSA level & $<0.001$ & 1.032 & 1.026 & 1.037 & $<0.001$ & 1.018 & 1.011 & 1.026 \\
\hline Gleason score & $<0.001$ & 2.152 & 1.932 & 2.398 & $<0.001$ & 1.690 & 1.484 & 1.925 \\
\hline Margin status & $<0.001$ & 3.136 & 2.499 & 3.935 & $<0.001$ & 2.146 & 1.644 & 2.800 \\
\hline pTNM & $<0.001$ & 3.279 & 2.706 & 3.973 & 0.001 & 1.544 & 1.207 & 1.974 \\
\hline CCN3 low/high & 0.013 & 1.400 & 1.072 & 1.828 & 0.010 & 1.437 & 1.092 & 1.890 \\
\hline \multicolumn{9}{|l|}{5 Years } \\
\hline Preoperative PSA level & $<0.001$ & 1.032 & 1.028 & 1.037 & $<0.001$ & 1.020 & 1.013 & 1.026 \\
\hline Gleason score & $<0.001$ & 2.107 & 1.916 & 2.317 & $<0.001$ & 1.698 & 1.517 & 1.900 \\
\hline Margin status & $<0.001$ & 2.590 & 2.126 & 3.155 & $<0.001$ & 1.740 & 1.383 & 2.190 \\
\hline pTNM & $<0.001$ & 3.108 & 2.623 & 3.684 & $<0.001$ & 1.563 & 1.260 & 1.939 \\
\hline
\end{tabular}

BCR, biochemical relapse; CPCBN, Canadian Prostate Cancer Biomarker Network; PSA, prostate-specific antigen; pTNM, primary tumor, node, and metastases; TMA, tissue microarray. 
Table 3 Cox Regression Analysis on the TF123 and CPCBN TMA Series for Bone Metastasis as End Point

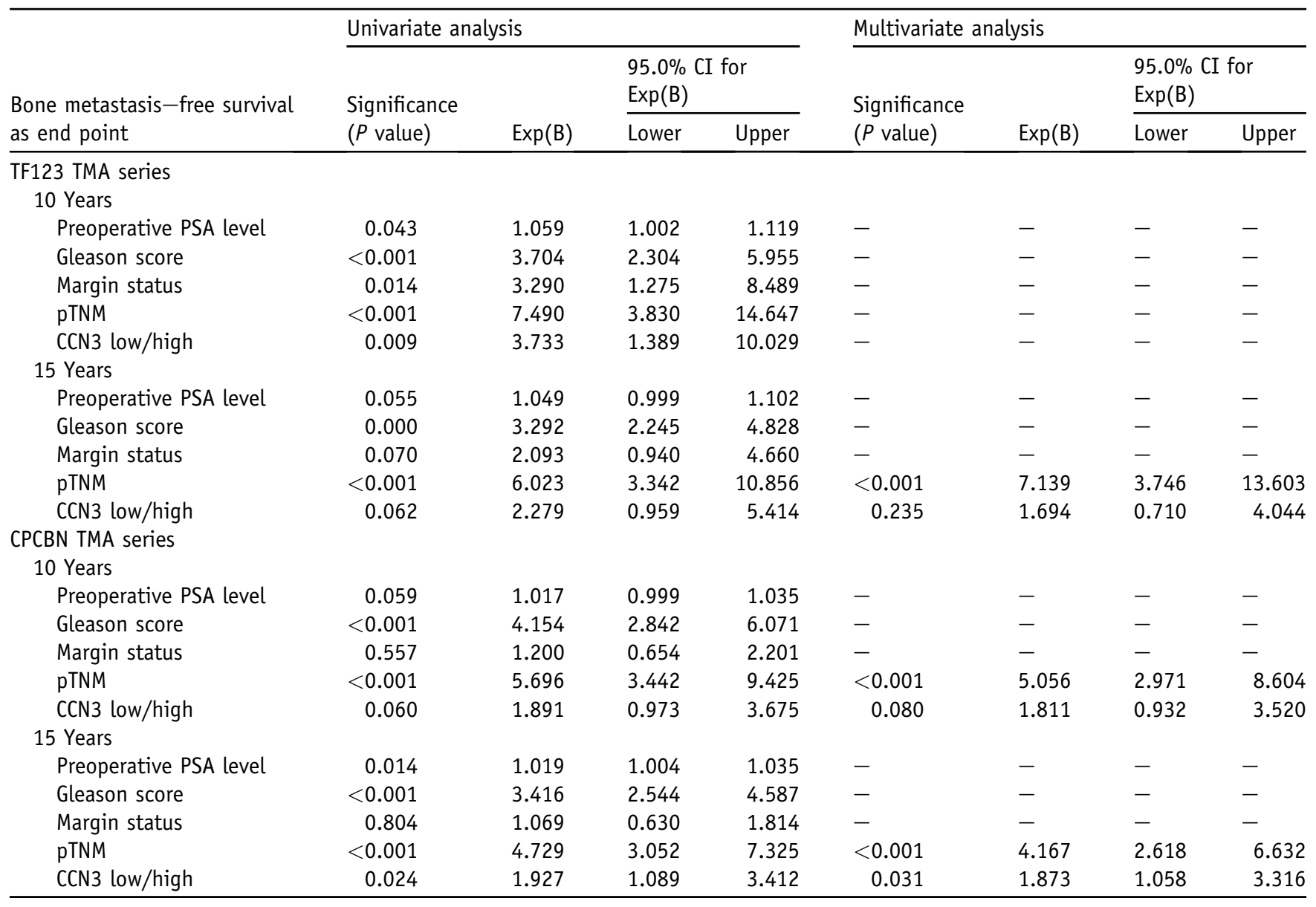

-, multivariate analysis was not performed; CPCBN, Canadian Prostate Cancer Biomarker Network; PSA, prostate-specific antigen; pTNM, primary tumor, node, and metastases; TMA, tissue microarray.

$1.092-1.890)$; and 5 years: $P=0.006 ; \operatorname{Exp}(\mathrm{B})=1.401(95 \%$ CI, 1.100-1.784)] (Table 2). A high level of CCN3 trends toward association with the development of bone metastasis at 10 years $[P=0.060 ; \operatorname{Exp}(\mathrm{B})=1.891(95 \% \mathrm{CI}$, $0.973-3.675)]$, although this association was significant at 15 years $[P=0.024 ; \operatorname{Exp}(\mathrm{B})=1.927(95 \% \mathrm{CI}, 1.089-3.412)]$ and remains independent in multivariate analyses $[P=0.031$; $\operatorname{Exp}(\mathrm{B})=1.873(95 \% \mathrm{CI}, 1.058-3.316)]$ (Table 3). PCspecific overall survival was not different between CCN3high and CCN3-low expressing patients in the CPCBN cohort in Kaplan-Meier analysis (Supplemental Figure S6C) or in a Cox model $[P=0.253 ; \operatorname{Exp}(\mathrm{B})=1.527(95 \% \mathrm{CI}$, 0.739-3.153)].

Together, these analyses show that high $\mathrm{CCN} 3$ expression is not associated with tumor suppression in prostate cancer and may be associated with aggressive disease characteristics.

\section{Discussion}

CCN3 has previously been proposed to act as a functional mediator of bone metastasis from both breast and prostate cancer through cell intrinsic mechanisms and cross talk with the bone microenvironment. ${ }^{10-12,16}$ However, recent studies have suggested that $\mathrm{CCN} 3$ is a tumor suppressor in PC cells, and that $\mathrm{AR}$ and enhancer of zeste homolog 2 (EZH2) signaling suppress CCN3 expression. ${ }^{6,7}$ Furthermore, CCN3 can functionally antagonize AR signaling. ${ }^{6}$ This study aimed to reconcile whether $\mathrm{CCN} 3$ behaves like a tumor suppressor or promotes aggressive PC using preclinical animal models and assessing $>1500$ human PC specimens in tissue microarrays. These results support a role for $\mathrm{CCN} 3$ as a functional mediator of $\mathrm{PC}$ aggressiveness and are not consistent with its proposed role as a tumor suppressor. It has been suggested that $\mathrm{CCN} 3$ can exert tumor suppressor activities when retained intracellularly, which contrasts its functional roles as a secreted protein in the tumor microenvironment. ${ }^{6}$ These studies have demonstrated that an N-terminal truncation of $\mathrm{CCN} 3$ can translocate to the nucleus, where it functions as a transcriptional repressor. ${ }^{6}$ However, such variants of CCN3 have yet to be identified in human specimens, making the relevance of this finding unclear. In addition, in the $\mathrm{LNCaP}$ C4-2 overexpression system used in this study, substantial CCN3 protein in whole cell lysates was not observed (data not 
A

Q1
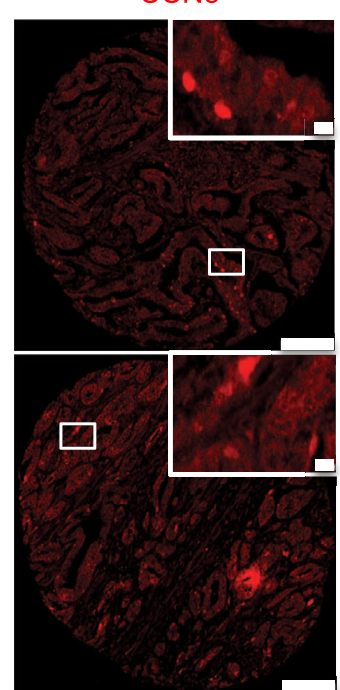

Q2

Q3
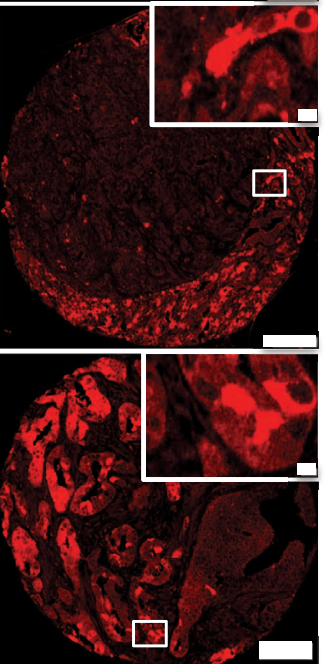

B

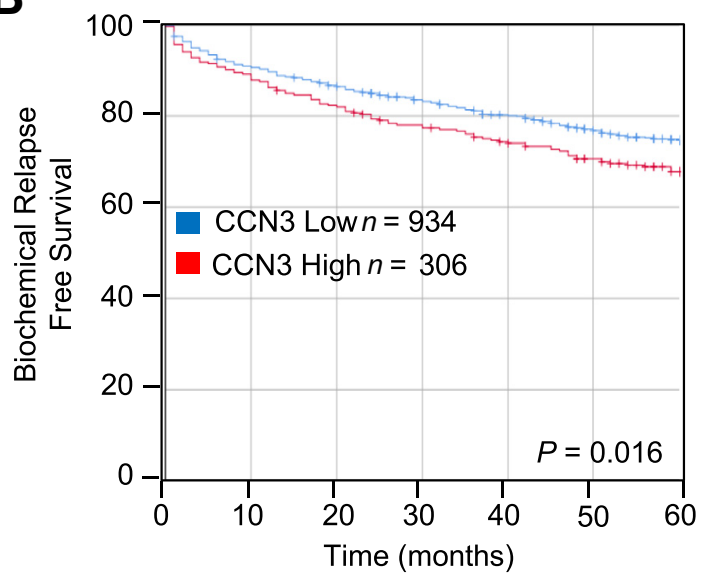

DAPI/CCN3
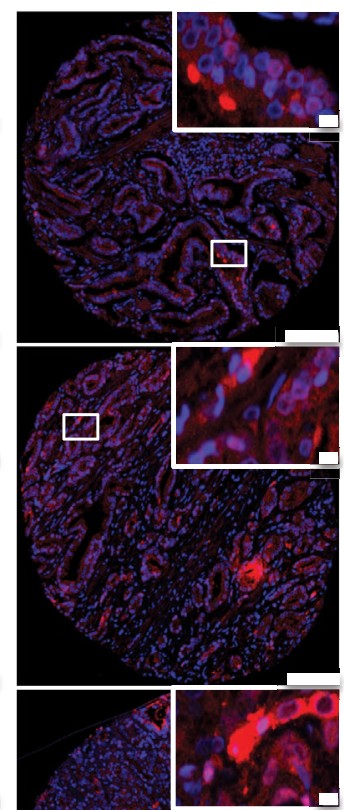

[6]

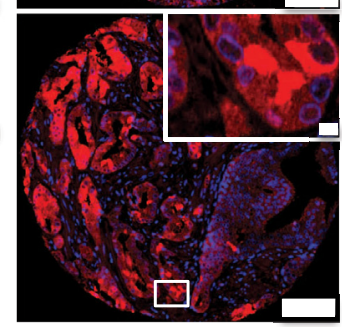

C

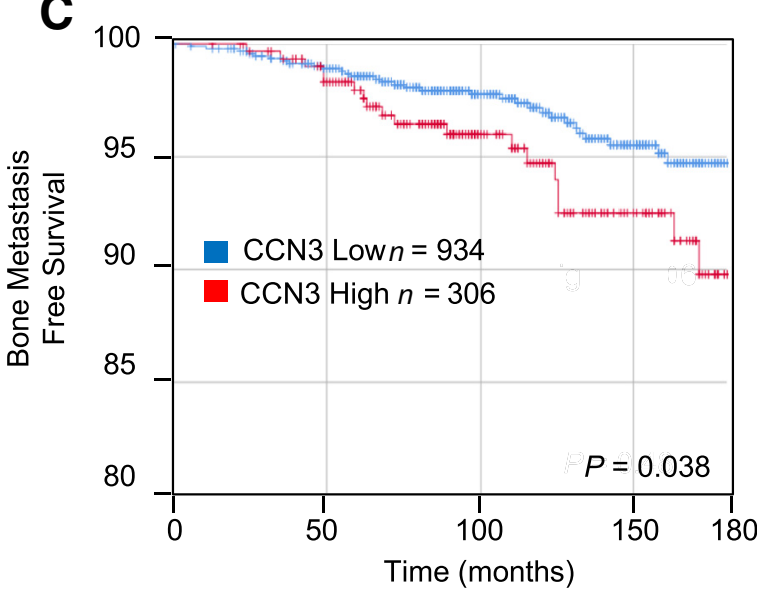

Figure 3 High CCN3 expression by immunohistofluorescence (IHF) correlates with biochemical relapse and bone metastasis in Canadian Prostate Cancer Biomarker Network (CPCBN) tissue microarray (TMA). A: The CPCBN TMA was assessed for CCN3 expression (Ab137677) by IHF, with simultaneous IHF of cytokeratins (CKs) 8 and 18 and DAPI staining. CCN3 high is classified as tumors in the fourth quartile ( $Q$ ) of expression, whereas CCN3 low comprises quartiles 1, 2, and 3. Representative images demonstrating staining intensities of each quartile are shown. Boxed areas are shown at a higher magnification in the insets. B: CCN3 high expression is correlated with decreased biochemical relapse-free survival at 5 years of follow-up $(P=0.016)$. C: Bone metastasis-free survival at 15 years of follow-up $(P=0.038) . n=1259$ primary prostate cancer patient samples $(\mathbf{A}-\mathbf{C}) ; n=306$ (CCN3 high); $n=934$ (CCN3 low). Scale bars: $100 \mu \mathrm{m}$ (A, main images); $5 \mu \mathrm{m}$ (A, insets). 


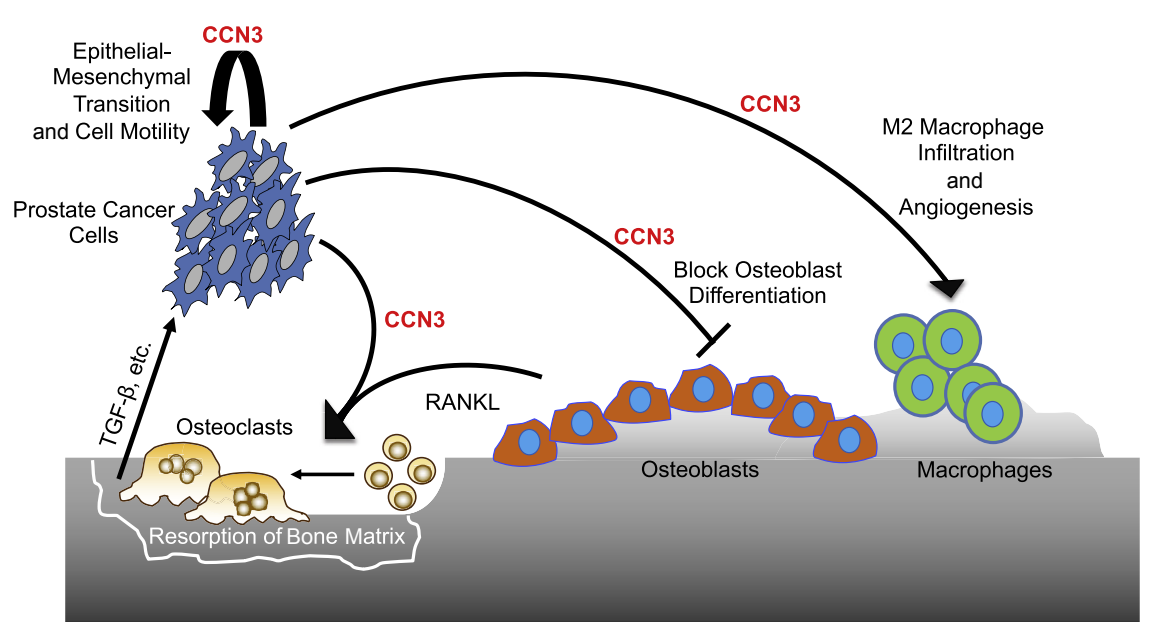

Figure 4 Schematic depicting the multifaceted roles of $\mathrm{CCN} 3$ in prostate cancer bone metastasis. CCN3 expression in prostate cancer cells has been proposed to promote metastasis via inducing epithelial-mesenchymal transition and prostate cancer cell motility through expression of intercellular adhesion molecule 1 . In addition, CCN3 promotes osteoclastogenesis and blocks osteoblast differentiation, resulting in net resorption of bone matrix and release of stored growth factors, such as transforming growth factor (TGF)- $\beta$, which feed back onto cancer cells, enhancing their proliferation and invasive capacity. Secreted CCN3 also has been reported to promote infiltration of M2 macrophages, resulting in increased angiogenesis. RANKL, receptor activator of nuclear factor kappa-B ligand.

shown), whereas CCN3 was secreted into the conditioned media at high levels. Furthermore, in CCN3-overexpressing LNCaP C4-2 cells, direct inhibition of AR expression or its subcellular relocalization was absent. Finally, suppression of PSA, an AR transcriptional target, was not observed in CCN3-overexpressing prostate cancer cells.

Overexpression of CCN3 in LNCaP C4-2 cells exerts no effect on tumor cell intrinsic proliferative, migratory, or invasive ability. However, when inoculated in immunecompromised mice by intracardiac injection, $\mathrm{LNCaP} \mathrm{C} 4-2$ cells overexpressing $\mathrm{CCN} 3$ form more frequent bone metastases and bone metastases that demonstrate a distinctly osteolytic phenotype compared with vector control-expressing cells. Because of the lack of an observed tumor cell intrinsic phenotype on proliferation, migration, and invasion using in vitro assays, we postulate that the enhanced ability to form bone metastases conferred by CCN3 is caused by interactions with the bone microenvironment. Mechanistically, in the context of breast cancer, a pro-bone metastatic phenotype induced by $\mathrm{CCN} 3$ involves modulation of the osteoprotegerin $(O P G)$ /receptor activator of nuclear factor kappa-B ligand (RANKL) axis to favor RANKLdependent osteoclastogenesis and increased bone resorption. ${ }^{11}$ Similarly, we demonstrate that CCN3 expression in LNCaP C4-2 PC cells, which normally form osteoblastic/ mixed lesions, ${ }^{24,25}$ promotes increased osteoclast density and bone resorption. More important, these findings were extended on by demonstrating that the osteolytic effects enabled by $\mathrm{CCN} 3$ expression are abolished when the $\mathrm{CT}$ domain of $\mathrm{CCN} 3$ is deleted. This implies a functional role for the CT domain in promoting osteoclastogenesis and/or inhibiting osteoblast differentiation. The CCN3 CT domain is known to bind a variety of extracellular proteins, such as platelet-derived growth factor, Notch1, and transforming growth factor- $\beta .^{5}$ Transforming growth factor- $\beta$ is a widely recognized mediator of osteolytic bone metastasis; thus, it is plausible that $\mathrm{CCN} 3$ binding to transforming growth factor- $\beta$ in the bone microenvironment can augment this function. ${ }^{28}$ This finding implies the importance of the CCN3 splice variants expressed in a particular tumor, given that $\mathrm{CCN}$ proteins lacking the $\mathrm{CT}$ domain have been previously identified in human specimens. ${ }^{29} \mathrm{~A}$ schematic outlining the potential roles played by $\mathrm{CCN} 3$ in promoting prostate cancer bone metastasis is shown in Figure 4.

Analysis of the CPCBN primary PC tissue microarray confirms that CCN3 is positively correlated with PC aggressiveness, which is not consistent with a tumor suppressor role for CCN3 in patients with PC. This finding is confirmed using an independent TMA (TF123) with an independent antibody and immunohistochemistry staining approaches instead of immunohistofluorescence. Together, these findings add important clinical data supporting a role for $\mathrm{CCN} 3$ as a functional mediator of $\mathrm{PC}$ bone metastasis. ${ }^{9-12,16}$ Interestingly, CCN3 expression inversely correlated with PSA expression, albeit weakly. This is in line with previously published data demonstrating that $\mathrm{CCN} 3$ inhibits $\mathrm{AR}$ signaling $^{6}$ and AR signaling represses $\mathrm{CCN} 3$ expression. ${ }^{7}$ Together, this work supports further investigation of $\mathrm{CCN} 3$ as a prognostic biomarker to predict $\mathrm{PC}$ recurrence to bone in prospective cohorts.

\section{Acknowledgments}

We thank the patients who donated the tissues studied in this work; the McGill/Goodman Cancer Research Centre Histology core facility, the McGill Comparative Medicine and Animal Resources Centre, and the McGill Centre for Bone and Periodontal Research for technical assistance; and the molecular pathology core facility of the Centre de Recherche du Centre Hospitalier de l'Université de Montréal for Ventana staining, image scanning, and image analysis; members of the Siegel laboratory for thoughtful discussions and critical reading of the manuscript; and the following individuals for contribution to the Canadian Prostate Cancer Biomarker Network: Dominique Trudel, Mathieu Latour, Nathalie Delvoye, Jean-Baptiste Lattouf, Pierre Karakiewicz, Armen Aprikian, Fadi Brimo, Simone 
Chevalier, Lucie Hamel, Eleonora Scarlata, Louis Lacombe, Alain Bergeron, Hélène Hovington, Hervé Brisson, Céline Veilleux, Neil E. Fleschner, Theodorus van der Kwast, Martin Gleave, and Ladan Fazli.

V.O., M.D., and P.M.S. conceived and designed the study; M.D., V.O., L.C., E.S., D.P., M.G.A., V.B., C.C., and P.M.S. developed methods; M.D., V.O., L.C., E.S., D.P., M.G.A., V.B., C.C., A.M.M.-M., F.S., and P.M.S. acquired data; V.O., M.D., L.C., E.S., D.P., M.G.A., V.B., C.C., and P.M.S. analyzed data; M.D., V.O., L.C., E.S., D.P., M.G.A., V.B., C.C., A.M.M.-M., F.S., and P.M.S. wrote, reviewed, and revised the manuscript; M.D., V.O., L.C., E.S., M.A., V.B., and C.C. provided administrative, technical, or material support; A.-M.M.-M., F.S., and P.M.S. supervised the study.

\section{Supplemental Data}

Supplemental material for this article can be found at http://doi.org/10.1016/j.ajpath.2019.04.006.

\section{References}

1. Gartrell BA, Saad F: Managing bone metastases and reducing skeletal related events in prostate cancer. Nat Rev Clin Oncol 2014, 11: 335-345

2. Chang AJ, Autio KA, Roach M 3rd,, Scher HI: High-risk prostate cancer: classification and therapy. Nat Rev Clin Oncol 2014, 11: 308-323

3. Brigstock DR, Goldschmeding R, Katsube KI, Lam SC, Lau LF, Lyons K, Naus C, Perbal B, Riser B, Takigawa M, Yeger H: Proposal for a unified CCN nomenclature. Mol Pathol 2003, 56:127-128

4. Li J, Ye L, Owen S, Weeks HP, Zhang Z, Jiang WG: Emerging role of $\mathrm{CCN}$ family proteins in tumorigenesis and cancer metastasis (review). Int J Mol Med 2015, 36:1451-1463

5. Ouellet V, Siegel PM: CCN3 modulates bone turnover and is a novel regulator of skeletal metastasis. J Cell Commun Signal 2012, 6:73-85

6. Fong KW, Zhao JC, Kim J, Li S, Yang YA, Song B, Rittie L, Hu M, Yang X, Perbal B, Yu J: Polycomb-mediated disruption of an androgen receptor feedback loop drives castration-resistant prostate cancer. Cancer Res 2017, 77:412-422

7. Wu L, Runkle C, Jin HJ, Yu J, Li J, Yang X, Kuzel T, Lee C, Yu J: $\mathrm{CCN} 3 / \mathrm{NOV}$ gene expression in human prostate cancer is directly suppressed by the androgen receptor. Oncogene 2014, 33:504-513

8. Maillard M, Cadot B, Ball RY, Sethia K, Edwards DR, Perbal B, Tatoud R: Differential expression of the ccn3 (nov) proto-oncogene in human prostate cell lines and tissues. Mol Pathol 2001, 54:275-280

9. Chen PC, Lin TH, Cheng HC, Tang CH: CCN3 increases cell motility and ICAM-1 expression in prostate cancer cells. Carcinogenesis 2012, 33:937-945

10. Chen PC, Tai HC, Lin TH, Wang SW, Lin CY, Chao CC, Yu HJ, Tsai YC, Lai YW, Lin CW, Tang CH: CCN3 promotes epithelialmesenchymal transition in prostate cancer via FAK/Akt/HIF-1alphainduced twist expression. Oncotarget 2017, 8:74506-74518

11. Chen PC, Cheng HC, Tang $\mathrm{CH}$ : CCN3 promotes prostate cancer bone metastasis by modulating the tumor-bone microenvironment through RANKL-dependent pathway. Carcinogenesis 2013, 34:1669-1679

12. Chen PC, Cheng HC, Wang J, Wang SW, Tai HC, Lin CW, Tang CH: Prostate cancer-derived CCN3 induces M2 macrophage infiltration and contributes to angiogenesis in prostate cancer microenvironment. Oncotarget 2014, 5:1595-1608

13. Bleau AM, Planque N, Lazar N, Zambelli D, Ori A, Quan T, Fisher G, Scotlandi K, Perbal B: Antiproliferative activity of CCN3: involvement of the C-terminal module and post-translational regulation. J Cell Biochem 2007, 101:1475-1491

14. Katsuki Y, Sakamoto K, Minamizato T, Makino H, Umezawa A, Ikeda MA, Perbal B, Amagasa T, Yamaguchi A, Katsube K: Inhibitory effect of $\mathrm{CT}$ domain of $\mathrm{CCN} 3 / \mathrm{NOV}$ on proliferation and differentiation of osteogenic mesenchymal stem cells, Kusa-A1. Biochem Biophys Res Commun 2008, 368:808-814

15. Perbal B: Nuclear localisation of NOVH protein: a potential role for NOV in the regulation of gene expression. Mol Pathol 1999, 52:84-91

16. Ouellet V, Tiedemann K, Mourskaia A, Fong JE, Tran-Thanh D, Amir E, Clemons M, Perbal B, Komarova SV, Siegel PM: CCN3 impairs osteoblast and stimulates osteoclast differentiation to favor breast cancer metastasis to bone. Am J Pathol 2011, 178:2377-2388

17. Rose AA, Annis MG, Dong Z, Pepin F, Hallett M, Park M, Siegel PM: ADAM10 releases a soluble form of the GPNMB/Osteoactivin extracellular domain with angiogenic properties. PLoS One 2010, 5:e12093

18. Rose AA, Pepin F, Russo C, Abou Khalil JE, Hallett M, Siegel PM: Osteoactivin promotes breast cancer metastasis to bone. Mol Cancer Res 2007, 5:1001-1014

19. Ouellet V, Aprikian A, Bergeron A, Brimo F, Bristow RG, Chevalier S, Drachenberg D, Fazli L, Fleshner NE, Gleave M, Karakiewicz P, Klotz L, Lacombe L, Lattouf JB, van der Kwast T, Squire JA, Latour M, Trudel D, Mes-Masson AM, Saad F: The Terry Fox Research Institute Canadian Prostate Cancer Biomarker Network: an analysis of a pan-Canadian multi-center cohort for biomarker validation. BMC Urol 2018, 18:78

20. Tiedemann K, Sadvakassova G, Mikolajewicz N, Juhas M, Sabirova Z, Tabaries S, Gettemans J, Siegel PM, Komarova SV: Exosomal release of L-plastin by breast cancer cells facilitates metastatic bone osteolysis. Transl Oncol 2018, 12:462-474

21. Corey E, Quinn JE, Bladou F, Brown LG, Roudier MP, Brown JM, Buhler KR, Vessella RL: Establishment and characterization of osseous prostate cancer models: intra-tibial injection of human prostate cancer cells. Prostate 2002, 52:20-33

22. Corey E: Xenograft models of human prostate cancer. Prostate Cancer. Edited by Isaacs WB, Chung LWK, Simons JW. Totowa, NJ: Humana Press, 2007

23. Henry MD, Silva MD, Wen S, Siebert E, Solin E, Chandra S, Worland PJ: Spiculated periosteal response induced by intraosseous injection of 22Rv1 prostate cancer cells resembles subset of bone metastases in prostate cancer patients. Prostate 2005, 65:347-354

24. Thalmann GN, Anezinis PE, Chang SM, Zhau HE, Kim EE, Hopwood VL, Pathak S, von Eschenbach AC, Chung LW: Androgenindependent cancer progression and bone metastasis in the $\mathrm{LNCaP}$ model of human prostate cancer. Cancer Res 1994, 54:2577-2581

25. Thalmann GN, Sikes RA, Wu TT, Degeorges A, Chang SM, Ozen M, Pathak S, Chung LW: LNCaP progression model of human prostate cancer: androgen-independence and osseous metastasis. Prostate 2000, 44:91-103

26. Wu TT, Sikes RA, Cui Q, Thalmann GN, Kao C, Murphy CF, Yang H, Zhau HE, Balian G, Chung LW: Establishing human prostate cancer cell xenografts in bone: induction of osteoblastic reaction by prostate-specific antigen-producing tumors in athymic and SCID/bg mice using LNCaP and lineage-derived metastatic sublines. Int J Cancer 1998, 77:887-894

27. Kim J, Coetzee GA: Prostate specific antigen gene regulation by androgen receptor. J Cell Biochem 2004, 93:233-241

28. Kang Y, Siegel PM, Shu W, Drobnjak M, Kakonen SM, CordonCardo C, Guise TA, Massague J: A multigenic program mediating breast cancer metastasis to bone. Cancer Cell 2003, 3:537-549

29. Perbal B: Alternative splicing of CCN mRNAs ... it has been upon us. J Cell Commun Signal 2009, 3:153-157 